\title{
Use of triamcinolone during vitrectomy surgery to visualize membranes and vitreous
}

\author{
Steven M Couch \\ Sophie J Bakri \\ Mayo Clinic Department \\ of Ophthalmology, Mayo Clinic, \\ Rochester, MN, USA
}

\begin{abstract}
Triamcinolone is a corticosteroid that is used to treat many ophthalmic diseases. Recently, its use has been advocated to aid in visualization of transparent tissue in ophthalmic surgery. It can be used in anterior segment surgery to help manage vitreous loss during complicated cataract surgery. It has been used to visualize the posterior cortical vitreous during pars plana vitrectomy. In addition, it can be useful in the visualization and peeling of the internal limiting membrane. Triamcinolone has been advocated in surgical repair of proliferative vitreoretinopathy both for its visualizing properties and its anti-inflammatory properties. Overall, triamcinolone use in surgery is safe with low incidence of complications including elevated intraocular pressure, cataract and endophthalmitis.
\end{abstract}

Keywords: cataract surgery, chromovitrectomy, epiretinal membrane, internal limiting membrane, triamcinolone acetonide, vitrectomy

Triamcinolone acetonide (TA) is a synthetic corticosteroid that has many uses is medicine. It can be administered in a multitude of ways including orally, parenterally, topically, by inhalation and intraocularly. While TA has been used in the treatment of ophthalmic diseases for many decades, it has recently been advocated as an adjuvant in ophthalmic surgery. The main use of TA in ophthalmic surgery is to aid in visualization of transparent membranes within the eye.

Triamcinolone is a corticosteroid that is well known for its anti-inflammatory, anti-permeability and anti-fibrotic properties, which justifies its use in inflammatory and vascular ophthalmic diseases (Floman and Zor 1977; Lewis et al 1986). However, recent evidence suggests that this water-insoluble steroid is also useful for ophthalmic surgery because it aids in visualization of ophthalmic structures, such as vitreous and internal limiting membrane. This property is heavily reliant upon the insoluble nature of the white crystals and the integration of these crystals into loosely organized collagen matrices. These crystals are thought to be interwoven into the collagen bundles of the vitreous (Peyman et al 2000; Enaida et al 2003). An extension of this mechanism is thought to be responsible for the staining of the superficial portions of an internal limiting membrane (ILM) and an epiretinal membrane (ERM). Studies have shown by electron microscopy that after manual vitreous detachment a small layer of cells and collagen fibers remain attached to the retina. This residual material may be responsible for the integration of TA crystals, which allow the visualization of the ILM and ERM (Shah et al 2004; Horio et al 2005; Tognetto et al 2005). Since no known biochemical reaction occurs to cause this visualization, the TA can be washed away with careful and continuous irrigation or aspiration after instillation in the eye.

Intraocular TA can be prepared in several ways. Triesence ${ }^{\circledR}$ (Alcon, Fort Worth, TX, USA) is a preservative-free preparation of TA that is approved by the FDA for intraocular use including use for vitreous visualization in intraocular surgery. It comes as a $1 \mathrm{~mL}$ vial 
containing a suspension of TA with concentration of $40 \mathrm{mg} / \mathrm{mL}$. Recommended dosing is $1-4 \mathrm{mg}(25-100 \mu \mathrm{L})$ administered intravitreally. Trivaris ${ }^{\circledR}$ (Allergan, Irvine, CA, USA) was recently approved as a preservative-free gel for intravitreal injection. It comes in a single-use syringe with $8 \mathrm{mg}(80 \mathrm{mg} / \mathrm{mL})$ of TA suspension. As an off-label use, Kenalog ${ }^{\circledR}$ (BristolMyers Squibb, Peapack, NJ, USA) can be administered for the same indication. Kenalog ${ }^{\circledR}$ contains benzyl alcohol as a preservative; many retinal surgeons prefer to use non-preserved Kenalog $^{\circledR}$, after a compounding pharmacy removes the preservative (Bakri et al 2005). The standard concentration of this preparation is $40 \mathrm{mg} / \mathrm{mL}$. The triamcinolone is often diluted with balanced salt solution at about 1:4 before instilling in the eye during vitrectomy.

Chromovitrectomy is the use of chemicals to stain semitransparent preretinal structures as an aid in successful vitreoretinal surgery. Several different dyes have been tried, and only a few substances remain as commonplace in surgery including indocyanine green (ICG), trypan blue (TB) and recently TA. ICG is a water-soluble dye that is used in vitrectomy because it binds to type IV collagen, allowing visualization of the ILM. ICG is well known for its use in retinochoroidal angiography. TB is also a water-soluble dye, but it has a high affinity for cellular components, which allows visualization of an ERM (Rodrigues et al 2005). As discussed above, TA intertwines itself in collagen matrices and will therefore coat both an ERM and ILM (Shah et al 2004; Horio et al 2005; Tognetto et al 2005). When comparing TA and ICG, there are many reasons to favor TA. First, unlike ICG, which requires a specific dilution ratio, TA does not require any preparation in the operating room. ICG, unlike TA, cannot be completely removed from all the tissues in the eye after it is used, as it stains the tissue. Triamcinolone coats the tissue. TA is less expensive than ICG (US \$25 per dose versus US \$135 per dose). Multiple adverse effects have been linked to ICG, including retinal pigment epithelial defects, optic nerve damage, long-term retinal staining, and photosensitivity to retinal laser after administration (Rodrigues et al 2005; Gandorfer et al 2003). Some studies have indicated that visual outcomes are worse after ICG-assisted ILM peeling (Rodrigues et al 2005). However, as described earlier, ICG stains the ILM directly, unlike TA, which does not differentiate between ILM and ERM because it simply coats the surface. TB possibly has some retinal toxic effects described in animal studies (Veckeneer et al 2001). Since TA simply integrates into the collagen matrix it can be removed with continuous aspiration or irrigation. However, unlike ICG or TB, TA can be repeated until desired visualization is achieved (Shah et al 2004; Horio et al 2005; Rodrigues et al 2005; Kampougeris et al 2007).

\section{Surgical uses}

During cataract surgery, posterior capsule violation and prolapse of vitreous body are troublesome complications. Unless effectively treated vitreous loss may be a harbinger to poor intraoperative or post-operative outcomes from sequelae such as retinal detachment and macular edema. The incidence of these complications can be lessened with meticulous removal of all vitreous tissue within the anterior chamber (Arbisser et al 2006; Chang et al 2006). Localization of vitreous and knowing when the anterior vitrectomy is complete are difficult because of the invisible nature of vitreous. Burk et al (2003) first described the use of TA to aid in the visualization of lost vitreous. When TA suspension is irrigated into an anterior chamber containing vitreous, the white crystals mix into the matrix effectively highlighting the vitreous boundaries. Effective visualization of anterior chamber vitreous allows for thorough anterior vitrectomy and allows the surgeon to avoid maneuvers that increase vitreous traction. In addition, it also offers a comfortable endpoint to the anterior vitrectomy (Burk et al 2003; Arbisser et al 2006; Chang et al 2006; Yamakiri et al 2006).

Pars plana vitrectomy (PPV) is a common vitreoretinal procedure used in the treatment of multiple conditions including vitreomacular traction syndrome, macular hole, epiretinal membranes, advanced diabetic retinopathy, and retinal detachment (RD) repair. During PPV, thorough removal of the vitreous and the posterior hyaloid membrane is an important surgical goal. Vitreoretinal adhesion has been suspected in the evolution of complicated retinal detachments and severe diabetic retinopathy (Peyman et al 2000; Sakamoto et al 2002; Sonoda et al 2004; Doi et al 2005; Matsumo et al 2007). During vitrectomy a posterior vitreous separation is induced, which may be difficult in some patients, especially diabetics and young patients. Post-operative residual hyaloid may provide scaffolding for fibrovascular proliferation in proliferative diabetic retinopathy or may provide a foundation of traction, which may cause or exacerbate conditions like macular holes or vitreomacular traction syndrome (Sonoda et al 2004; Doi et al 2005). Meticulous removal of minute amounts of vitreous and hyaloid membrane requires patience and careful observation, but if the vitreous boundaries could be highlighted this process could be more efficient and effective (Figures 1 and 2). Peyman et al (2000) were the first to 


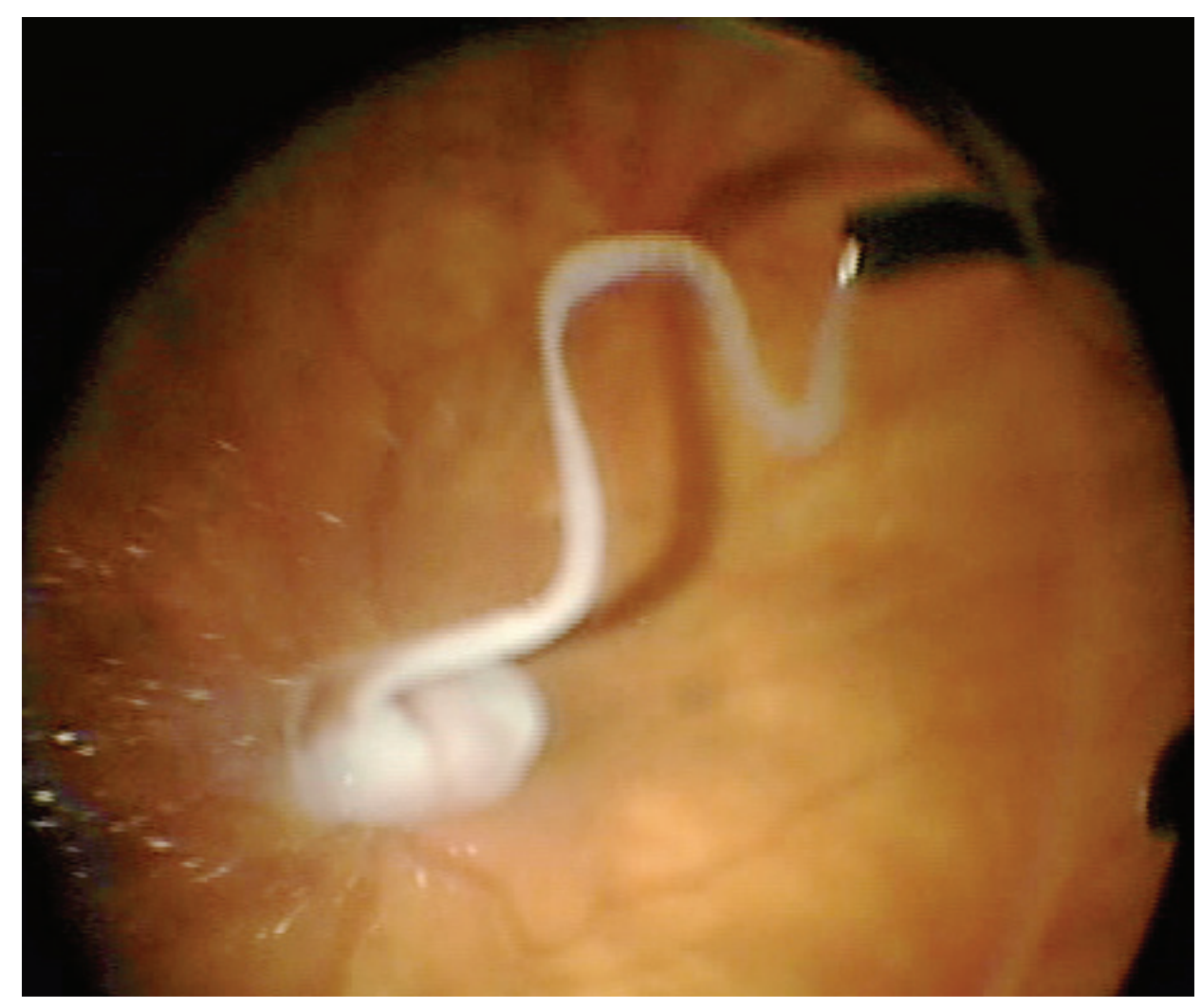

Figure I Injection of triamcinolone during vitrectomy.

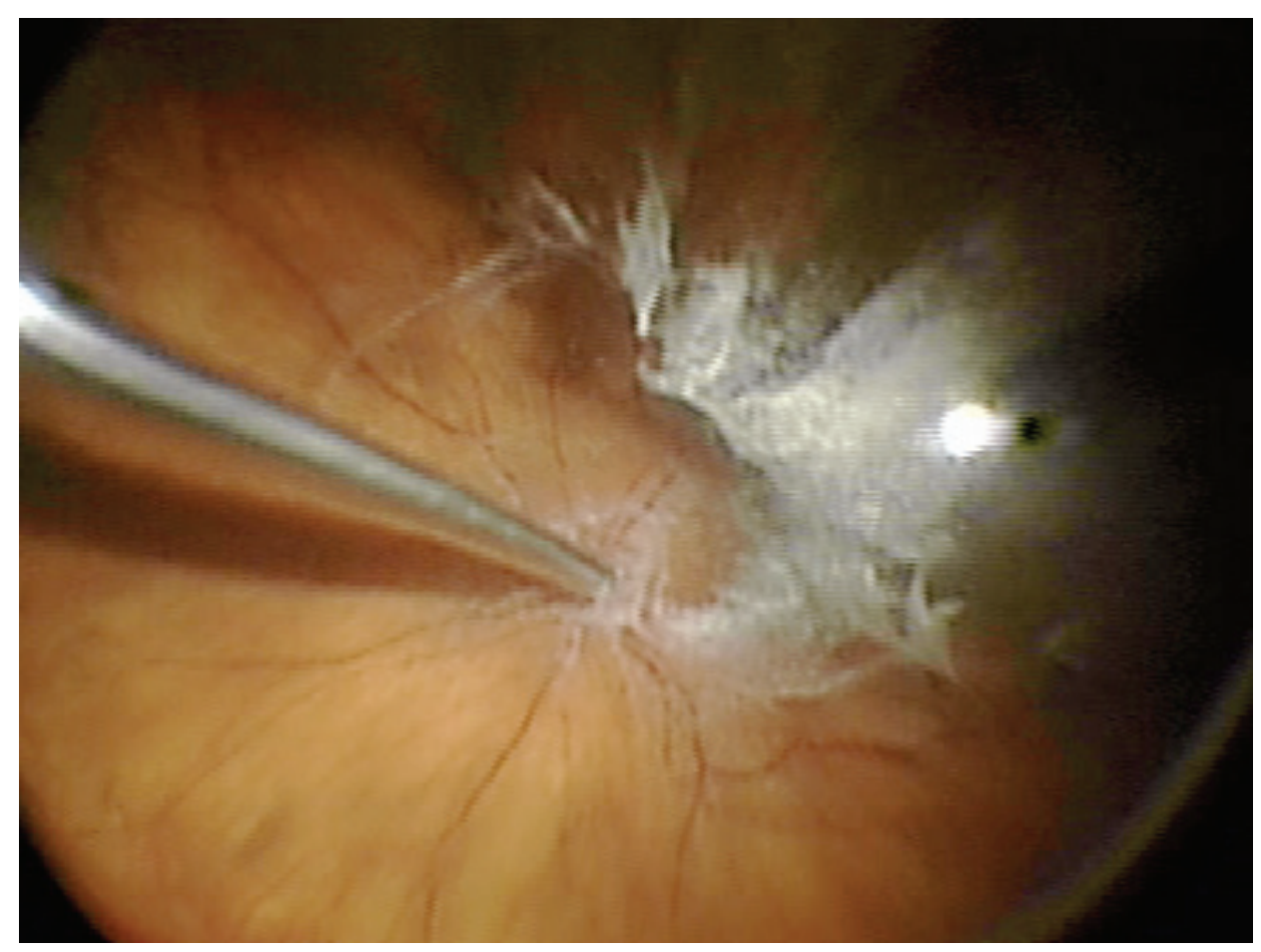

Figure 2 Triamcinolone staining of residual vitreous cortex. 
describe the use of TA to aid in vitreous visualization. It was noted that when TA is injected into the vitreous cavity the crystals become trapped in the vitreous gel and the suspension allows for good contrast between the whitened vitreous and the post-hyaloid space which harbors a free-floating suspension of TA particles. Because of better visualization, TA assistance in PPV has been shown to help surgeons perform a more thorough removal of vitreous and posterior hyaloid.

In addition to a comprehensive PPV, removal of the ILM is used as an adjunct in vitreoretinal surgery in the repair of macular holes, cellophane maculopathy, and macular edema. It is thought that peeling of the internal limiting membrane reduces tangential retinal traction and improves macular hole closure rate (Doi et al 2005). Removal of this transparent thin membrane proves difficult even for experienced surgeons. As described above, dyes have been utilized to aid in the visualization of the ILM and ERM. Injection of TA allows delineation of an ILM and ERM secondary to integration of the crystals into its superficial collagen fibrils (Figure 3) (Horio et al 2005). TA provides contrast between the highlighted ILM and ERM and those areas that have already been delaminated because the TA will not stain exposed retina (Shah et al 2004, 2005; Fraser et al 2003; Kimura et al 2004; Tognetto et al 2005). If TA is utilized in the vitrectomy stage of the procedure, it can be reinjected for ILM or ERM visualization and the surgeon may require reinjection if the TA is washed away by irrigation or aspiration. However, because of the TA crystals water insolubility, there have been some concerns with residual TA affecting macular hole closure rates, but visual outcomes are the same or better after the procedure (Karacorlu et al 2005; Kumagai et al 2007; Hikichi et al 2008).

One of the most common reasons for failure of $\mathrm{RD}$ repair is proliferative vitreoretinopathy (PVR). PVR is proliferation and subsequent contraction of a fibrous nonvascular epiretinal membrane at the vitreoretinal interface generally stimulated by rhegmatogenous retinal detachment (Girard et al 1994; Furino et al 2003). Subsequent surgery for RD with PVR is tedious and problematic as it is generally associated with poor anatomic and visual outcomes. One of the established treatments for PVR is PPV with the surgical goal to remove any residual vitreous and tractional ERMs. Removal of the ERM is thought to be the most important factor influencing clinical outcomes after surgery for PVR (Aaberg 1988; Abrams et al 1997; Girard et al 2004; Ueno et al 2007).

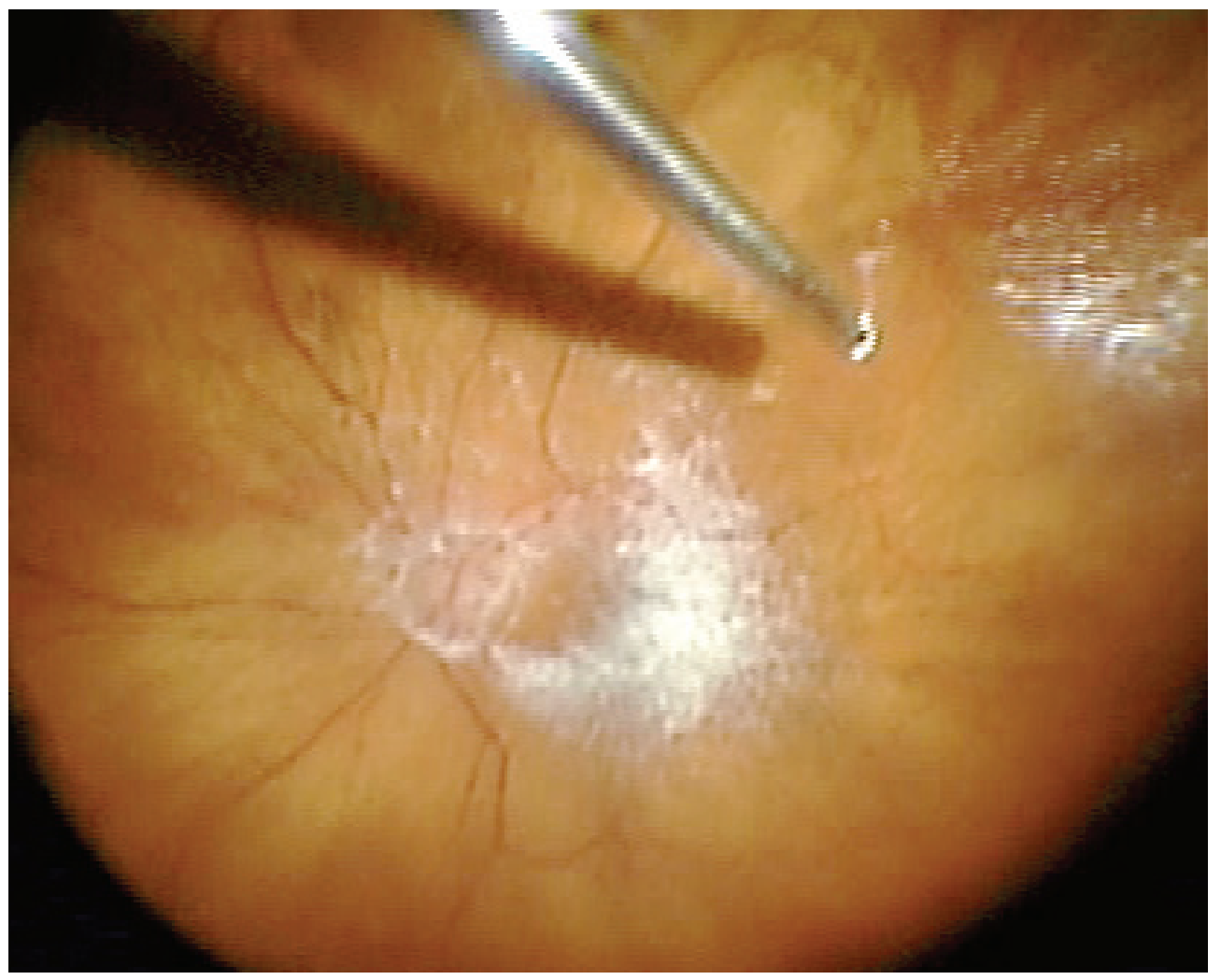

Figure 3 Enhanced visibility of epiretinal membrane with triamcinolone. 
As described previously, remaining posterior hyaloid face and ERMs are transparent making their removal tedious. TA has been used as an adjunct in visualization and subsequent surgical removal of these membranes and the remaining vitreous cortex. Previous studies have shown that intravitreal injection of corticosteroids, including TA, can be used in the treatment and prevention of further fibrous growth (Jonas et al 2000; Girard et al 2004). The anti-inflammatory and anti-fibrotic effects of TA may further advocate its use with this type of surgery. Several studies have advocated the use of TA in surgery for PVR with good success rates (Furino et al 2003; Ueno et al 2007). Sonada et al (2003) supported the use of TA assistance in PVR treatment associated with refractory uveitis with good results. Because of other surgical techniques used in the treatment of PVR, it is important to remember that silicone oil protracts the absorption of TA (Girard et al 2004).

Perfluorcarbon (PFC) liquid has been widely used in combination with PPV. However, because of its retinotoxic properties if left in the eye long term, it must be completely removed during vitrectomy. Because PFC is invisible, residual bubbles are commonly found after surgery. Hirata et al (2005) showed in vivo and in vitro that PFC adsorbs the TA and highlights residual PFC for easier removal.

Intravitreal TA has been used for its anti-inflammatory properties for many decades in the treatment of various intraocular diseases. It is well known for its anti-angiogenic and anti-fibrotic properties, which can have a beneficial effect in the treatment of conditions commonly treated with intraocular surgery (Kampougeris et al 2007). One study objectively showed a decrease in postoperative blood-ocular barrier breakdown after TA-assisted PPV, especially for diabetic macular edema (Sakamoto et al 2002).

A low incidence of adverse outcomes has been found with TA-assisted intraocular surgery. Recent studies showed a decrease in intraoperative complications including retinal detachment and retinal breaks (Yamakiri et al 2007). However, 1-year data showed no difference in visual acuity, need for additional surgeries, and adverse events between TA-assisted PPV and conventional PPV (Yamakiri et al 2008). As shown with previous studies of intravitreal triamcinolone, the more common complications include cataract formation and elevated intraocular pressure. However, the incidence of these complications are less than expected (Enaida et al 2003; Sakamoto et al 2002; Chang et al 2006; Kampougeris et al 2007; Kumagai et al 2007; Ueno et al 2007; Hikichi et al 2008). Several studies have shown residual retinal TA after PPV. While no direct retinotoxic effects have been shown with TA, there is still concern about toxic effects of the TA's vehicle (Kampougeris et al 2007). Other studies have been concerned where the TA crystals anatomically reside if they are left behind and have shown no long term visual or anatomic consequence even if left in within the fovea (Takeuchi et al 2003; Fukukita et al 2007; Hikichi et al 2008). Finally, intravitreal TA in nonvitrectomized eyes is associated with a low incidence of endophthalmitis and the same would be expected after TAassisted vitrectomy. It also may mask many of the common clinical signs of endophthalmitis after intraocular surgery (Yamashita et al 2004). Despite these complications, TA is considered safe by most clinicians for assistance with intraocular surgery.

Triamcinolone acetate has been used in ocular therapeutics and its indications are expanding. Because it integrates into collagen matrices, it has been used to help delineate vitreous, internal limiting membranes, and epiretinal membranes. This has been useful in treatment of complicated cataract surgery, pars plana vitrectomy, and macular surgery. With a low incidence of adverse events, it is regarded as safe for use in assistance with intraocular surgery.

\section{Disclosures}

The authors report no conflicts of interest.

\section{References}

Aaberg TM. 1988. Management of anterior and posterior proliferative vitreoretinopathy: XLV Edward Jackson Memorial Lecture. Am J Ophthalmol, 106:519-32.

Abrams GW, Azen SP, McCuen BW II, et al; for the Silicone Study Group. 1997. Vitrectomy with silicone oil or long-acting gas in eyes with severe proliferative vitreoretinopathy: results of additional and long-term follow up: Silicone Study Report 11. Arch Ophthalmol, 115:335-44.

Arbisser LB, Charles S, Howcroft M, et al. 2006. Management of vitreous loss and dropped nucleus during cataract surgery. Ophthalmol Clin N Am, 19:495-506.

Bakri SJ, Shah A, Faulk NS, et al. 2005 Intravitreal preservative-free triamcinolone acetonide for the treatment of macular oedema. Eye, 19:686-8.

Burk SE, Da Mata AP, Snyder ME, et al. 2003. Visualizing vitreous using Kenalog suspension. J Cataract Refract Surg, 29:645-51.

Chang C, Chiang S, Chen C, et al. 2006. Clinical outcomes of combined sutureless vitrectomy with triamcinolone stain to manage vitreous loss resulting from posterior capsule rupture during phacoemulsification. $J$ Cataract Refract Surg, 32:2054-9.

Doi N, Uemura A, Nakao K, et al. 2005. Vitreomacular adhesion and the defect in the posterior vitreous cortex visualized by triamcinoloneassisted vitrectomy. Retina, 25:591-4.

Enaida H, Hata Y, Ueno A, et al. 2003. Possible benefits of triamcinoloneassisted pars plana vitrectomy for retinal diseases. Retina, 23:764-70.

Floman N, Zor U. 1977. Mechanism of steroid action in ocular inflammation: inhibition of prostaglandin production. Invest Ophthalmol, 16:69-73.

Fraser EA, Cheema RA, Roberts MA. 2003. Triamcinolone acetonideassisted peeling of the retinal internal limiting membrane for macular surgery. Retina, 23:883-4. 
Fukukita M, Sasoh M, Matsubara H, et al. 2007. Triamcinolone acetonide remaining on the fovea after successful macular hole closure. Retina, $27: 122-3$

Furino C, Ferrari TM, Boscia F, et al. 2003. Triamcinolone-assisted pars plana vitrectomy for proliferative vitreoretinopathy. Retina, 23:771-6.

Gandorfer A, Haritoglou C, Gandorfer A, et al. 2003. Retinal damage from indocyanine green in experimental macular surgery. Invest Ophthalmol Vis Sci, 44:316-23.

Girard P, Mimoun G, Karpouzas, et al. 1994. Clinical risk factors for proliferative vitreoretinopathy after retinal detachment surgery. Retina, 14:417-24.

Hikichi F, Furukawa Y, Ohtsuka H, et al. 2008. Improvement of visual acuity one-year after vitreous surgery in eyes with residual triamcinolone acetonide at the macular hole. Am J Ophthalmol, 145:267-72.

Hirata F, Tamura H, Ogura Y. 2005. Visualization of residual perfluorocarbon liquid using intravitreal triamcinolone acetonide. Ophthalmic Surg Lasers Imaging, 36:169-72.

Horio N, Horiguchi M, Yamamoto N. 2005. Triamcinolone-assisted internal limiting membrane peeling during idiopathic macular hole surgery. Arch Ophthalmol, 123:96-9.

Jonas JB, Hayler JK, Panda-Jones S. 2000. Intravitreal injection of crystalline cortisone as adjunctive treatment of proliferative vitreoretinopathy. Br J Ophthalmol, 84:1064-7.

Kampougeris G, Chemma R, McPherson R, et al. 2007. Safety of triamcinolone acetonide (TA)-assisted pars plana vitrectomy in macular hole surgery. Eye, 21:591-4.

Karacorlu M, Ozdemir H, Karacoriu SA. 2005. Does intravitreal triamcinolone acetonide-assisted peeling of the internal limiting membrane effect the outcome of macular hole surgery? Graefe's Arch Clin Exp Ophthalmol, 243:754-7.

Kimura H, Kuroda S, Nagata M. 2004. Triamcinolone acetonide-assited peeling of the internal limiting membrane. Am J Ophthalmol, 137:172-3.

Kumagai K, Furukawa M, Ogino N, et al. 2007. Long-term outcomes of macular hole surgery with triamcinolone acetonide-assisted internal limiting membrane peeling. Retina, 27:1249-54.

Lewis GD, Campbell WB, Johnson AR. 1986. Inhibition of prostaglandin synthesis by glucocorticoids in human endothelial cell. Endocrinology, 199:62-9.

Matsumo H, Yamanaka I, Hisatomi T, et al. 2007. Triamcinolone acetonideassisted pars plana vitrectomy improves residual posterior vitreous hyaloid removal. Retina, 27:174-9.

Peyman GA, Cheema R, Conway M, et al. 2000. Triamcinolone acetonide as an aid to visualization of vitreous and the posterior hyaloid during pars plana vitrectomy. Retina, 20:554-5.
Rodrigues EB, Meyer CH, Kroll P. 2005. Chromovitrectomy: a new field in vitreoretinal surgery. Graefe's Arch Clin Exp Ophthalmol, 243:291-3.

Sakamoto T, Miyazaki M, Hisatomi T, et al. 2002. Triamcinolone-assisted pars plana vitrectomy improves the surgical procedures and decreases the postoperative blood-ocular barrier breakdown. Graefe's Arch Clin Exp Ophthalmol, 240:423-9.

Shah GK, Rosenblatt BJ, Blinder KJ, et al. 2005. Triamcinolone-assisted internal limiting membrane peeling. Retina, 25:972-5.

Shah GK, Rosenblatt BJ, Smith M. 2004. Internal limiting membrane peeling using triamcinolone acetonide: histopathologic confirmation. Am J Ophthalmol, 138:656-7.

Sonoda K, Enaida H, Ueno A, et al. 2003. Pars plana vitrectomy assisted by triamcinolone acetonide for refractory uveitis: a case series study. Br J Ophthalmol, 87:1010-14.

Sonoda K, Sakamoto T, Enaida H, et al. 2004. Residual vitreous cortex after surgical posterior vitreous separation visualized by intravitreous triamcinolone acetonide. Am J Ophthalmol, 111:226-30.

Takeuchi M, Katagiri Y, Usui M. 2003. Residual triamcinolone acetonide in the macular hole after vitrectomy. Am J Ophthalmol, 136:1174-6.

Tognetto D, Zenoni S, Sanguinetti G, et al. 2005. Staining of the internal limiting membrane with intravitreal triamcinolone acetonide. Retina, 25:462-7.

Ueno A, Enaida A, Hata Y, et al. 2007. Long-term clinical outcomes and therapeutic benefits of triamcinolone-assisted pars plana vitrectomy for proliferative vitreoretinopathy: A case study. Eu J Ophthalmol, 7:392-8.

Veckeneer M, van Overdam K, Monzer J, et al. 2001. Ocular toxity study of trypan blue injected into the vitreous cavity of rabbit eyes. Graefes Arch Clin Exp Ophthalmol, 239:698-704.

Yamakiri K, Sakamoto T, Noda Y, et al. 2007. Reduced incidence of intraoperative complications in a multicenter controlled clinical trial of triamcinolone in vitrectomy. Ophthalmology, 114:189-296.

Yamakiri K, Sakamoto T, Noda Y, et al. 2008. One-year results of a multicenter controlled clinical trial of triamcinolone in pars plana vitrectomy. Graefes Arch Clin Exp Ophthalmol, 246:959-66.

Yamakiri K, Uchino E, Kimura K, et al. 2006. Intracameral triamcinolone helps to visualize and remove the vitreous body in anterior chamber in cataract surgery. J Cataract Refract Surg, 32:2054-9.

Yamashita T, Doi N, Sakamoto T. 2004. Weak symptoms of bacterial endophthalmitis after a triamcinolone acetonide-assisted pars plana vitrectomy. Graefe's Arch Clin Exp Ophthalmol, 242:679-81. 